\title{
TECHNOLOGY ASSESSMENT IN A GLOBALIZED WORLD
}

\author{
Constanze SCHERZ, Julia HAHN, Miltos LADIKAS \\ Institute for Technology Assessment and Systems Analysis (ITAS)
}

\begin{abstract}
:
The interaction between science and policy is affected by increasingly dissolving boundaries whereby a number of issues arise, such as, what should political advice for national parliaments consist of in the face of global socio-technical developments? The Internet of Things in relation to Big Data resulting in enormously fast analysis of huge amounts of data, accelerates the dissolution of boundaries between science, society and policy at global level. This type of "globalization" raises questions that concern very diverse and intimate areas of life, ranging from food to health to work automation. How can access to science and technology in very different societal settings can be enabled, while also accounting for global developments? The article deals with the assumption that global effects of science and technology as well as global challenges lead to an urgent need to develop methodologies for analyzing and also shaping these developments. Under the umbrella term "Global Technology Assessment" (Global TA) it is argued that the problem-oriented, interdisciplinary methodology of technology assessment offers a promising frame to deal with cultural and ethical questions in relation to emerging technologies. This framework is needed in order to be able to develop meaningful national comparisons, but also in order to be able to approach the analysis of common future challenges on equal footing.
\end{abstract}

Key words: global TA, ethics, culture, technology assessment

\section{INTRODUCTION}

In the research funding program Horizon 2020 of the European Commission, the grand societal challenges are described. They revolve around issues such as health, demographic change, food security, sustainable agriculture, bioeconomy, clean and efficient energy, green transport, climate action, environment, resource efficiency, and innovative and reflective societies. The global impact of these challenges is obvious and therefore, any approach to dealing with them must have a global perspective. It is a fact, not only in Europe but also worldwide, that science, technology and innovation (STI) is seen as the way forward to dealing with these challenges.

At the same, STI has worldwide impacts and this could create problems for policy decisions, when technological effects conflict with national policies that are primarily geared to promoting the interests of their own economy within their own society. Technology Assessment (TA) as problemoriented research reflects on these topics, increasingly often on its global impacts and the efforts needed to handle global challenges. TA aims at supporting society and policy making in understanding and managing societal problems resulting from scientific and technological developments. It has always been an approach aimed at offering advice and presenting ways to deal with societal pressing questions in the interrelation of technology and science. The European project "Technology Assessment in Europe; Between Met- hod and Impact" (2002-2003) created a common conceptual and methodological basis for European TA experts and developed the following definition:

"Technology assessment (TA) is a scientific, interactive and communicative process which aims to contribute to the formation of public and political opinion on societal aspects of science and technology" [16].

Grunwald on the other hand, focuses on the manifold character of TA: "TA provides knowledge, orientation, or procedures on how to cope with certain problems at the interface between technology and society but it is neither able nor legitimized to solve these problems" [5].

Against this background, the possibilities for a global TA are contemplated. For this purpose, it is specified in more detail why a global TA is needed. This demand results from the experiences that characterize the development of TA in recent decades. Therefore, some highlights on the history of TA are explained. In addition, the example of the project "Global Ethics in Science and Technology" (GEST) is described as a first global approach in TA. In conclusion, it is argued that a global TA can succeed if culture, ethics and engagement as well as the similarities of national values systems are taken into account.

\section{EVOLUTION OF (GLOBAL) TA}

To understand the global approach of TA it is helpful to understand the evolution of TA. The history of TA is closely linked to its 'invention' as a policy consulting method and 
its first institutionalizations show that the cradle of TA is the USA and Europe. Even the term Technology Assessment (TA) is a Western invention but its purpose and methodologies are far from a Western exclusivity. TA is increasingly universal in its scope since its topics are interlinked with scientific and technological developments that take place worldwide.

Although TA activities have been part of S\&T since the beginning, official TA (termed as such) was established fifty years ago, focusing on concrete predictions of technological consequences. The main aim of this first TA was to gain advanced knowledge on technology options in order to create better informed policy decisions. This was an "early warning" system that was central to the identity of TA as it was seen as the means to identify potential hazards and minimize their effects.

Two examples, the Office for Technology Assessment in the U.S. Congress and the Office of Technology Assessment at the German Bundestag (TAB), offers insights into the political and societal conditions under which TA institutions have been established in the past. Concepts of TA were early discussed in the United States in the late 1960s "when tensions flared between executive and the congressional branches of the federal government about access to technical and scientific advice" [15]. After years of debate about the conceivable methods and styles of advice, Congress created the "Office of Technology Assessment" (OTA) in 1972 in order to assist and support the legislatives "in the identification and consideration of existing and probable impacts of technological application (to ensure that) the consequences of technological applications be anticipated, understood, and considered in determination of public policy on existing and emerging national problems" [2]. OTA was the first and largest Parliamentary TA office, and its history has therefore often been studied [1, 7, 12]. Unfortunately, the inner-organizational structure of OTA was never fully established before OTA had to close its doors in 1995 after more than 20 years of operation. Nevertheless, OTA is still a role model for others. The same approach to institutionalize TA was taken up by European parliamentary TA institutions founded in the 1980s and 1990s.

OTA represented what came to be known as "classical TA". This is the type of TA whose functions are still valid within the TA discipline nowadays and which include the identification of impacts of technology, assertion of cause-and-effect relationships and the identification of alternative programs and options for action. This set the paradigm of TA as an information service, offering possibilities for activities but no prerogatives, in other words, to answer the what "can" be done instead of what "should" be done.

The other example is TA at the German Bundestag which is strongly connected with the TAB, operated by the Institute for Technology Assessment and Systems Analysis (ITAS/KIT) since 1990. In the 1970s, debates on the opportunities and risks of scientific and technological developments increased not only in Germany but in some Western European countries. Numerous problematic consequences for society and the environment raised awareness in the national Parliament of the need for early assessment of the deve- lopment and use of technology. The parliamentarians debated the opportunities, risks and potentials of designing new forms of technology and soon focused on the question of whether and how TA might be used in support of decision-making processes.

On August 29, 1990, after long and intense debate on TA and its institutionalization the German Bundestag signed the first contract with the Karlsruhe Nuclear Research Center for a three-year pilot phase and TAB was founded [9]. The TAB follows the organization model of "shared parliament-science involvement" and its work focuses solely on the German Bundestag. The last decades showed that the need for parliamentary advice on technological issues has increased rather than decreased: the number of committees initiating and debating TAB studies has grown [4].

The evolution of TA is closely linked to its claim to advise policy. But TA is also relevant in other societal debates such as public debates (participatory TA) and engineering processes (encouraging interdisciplinarity in the innovation process). It is no longer a question whether TA is undertaken when science and technology develop and its results are applied in real life. TA is evident one way or another since any kind of application is necessarily the result of an assessment. What matters is when is TA done in the S\&T development trajectory and how it is done. There is tremendous variety in answering and one should view culture, values and politics realities as the main parameters in the answers.

\section{GLOBAL TA APPROACH}

As described, developments in science and technology have different and far-reaching effects all over the world. These depend on national cultures, political traditions and scientific practices how the governance of S\&T is built. There is increasing evidence for a common understanding that the governance has to be more resilient also in terms of their global impact. Increasing demands for engagement in S\&T decision making processes is one indicator for this. As Jasanoff writes: "In thinking about research today, policy-makers and the public inevitably focus on the accountability of science. As the relations of science have become more pervasive, dynamic, and heterogeneous, concerns about the integrity of peer review have transmuted into demands for greater public involvement in assessing the costs and benefits, as well as the risks and uncertainties, of new technologies" [11]. Harmonizing S\&T with societal objectives is a challenge for all nations. Therefore, growing interest in the development and the implementation of engagement processes from various sides can be observed [10]. The global level of S\&T is contrasted by plural and culturally-specific approaches towards political decisions. Those approaches reflect the values of a country. S\&T policies reflect the specific values and ethical considerations of a certain context (in a certain country). But, in any setting dealing with S\&T some form of ethical reflection takes place, be it as professional philosopher or as participant in societal debates. In this way, ethics can be understood as "a common platform for deliberation and discussion of values in society that is based on perceptions of right and wrong, is 
influenced by cultural norms, and aims at informing policy making" [14].

For a global approach this definition is very useful since it takes cultural specifics into account and does not limit ethics to a certain discipline but as something which takes place in the public discourse and in social interactions. Of course, what form the ethical debates on, for example Man-Machine interactions, take in different countries can vary. But are there possibilities of a global understanding? "Global ethics is not a field of academic study, it is an activity: the attempt to agree on fundamental conditions for human flourishing and to actively secure them for all" [13]. The challenge is then to take the local, national specifics into account while striving towards a global level as well. For this it is necessary to look in-depth in ethical debates that play a role in national contexts, to scale them up to a wider level with the aim to define a common ground.

A comparative framework for ethical debates on S\&T in different countries was developed in the project "GEST: Global Ethics in Science and Technology" funded by the European Commission (Framework 7 program), based on the assumption that the "nature of expectations, tensions and conflicts will vary, not only in relation to the contents of particular fields of science and technology, but also according to particular socio-economic conditions, cultural contexts and values in the different global regions" [13]. With the framework communalities and differences in the discourses on specific S\&T as well as in regard to regions can be understood. With this it becomes possible to compare countries and technologies and how these are translated into policy. The overall aim in the project was to come to "more responsive and robust practices of anticipatory governance of science and technology" [13].

For the framework three content-related discourses are relevant: innovation, risk as well as power and control. Reflective ethics (the more professional level) and lay morality (the public discourses) are cross-cutting. They form the way the discourses on innovation, risk and power and control are perceived in ethical analyses as well as broader public debates. This way the similarities and differences between the discourses and their specific contexts become apparent [13]. The GEST project focused on the regions of India, China and Europe and on specific issues such as nanotechnology or food security.

The following findings of the GEST project can only be noted at this point. The incorporation of ethics in policy-making happens always in connection to culture and this means that, among other things, the following parameters must be taken into account: dominant values, history and official governmental structures. They all influence the expression and direction of ethics debates. At the same time, private concerns, whether business-related or not, influence ethics debates by promoting moral arguments over certain world-views and policy choices.

Ethics can be both 'formally' (e.g. through the official structures in the decision-making system) and 'informally' (public perspective on ethics expressed by lay people) expressed.

Ethics derives from the dominant values that are held dear by society at the time of the debate.
The historical and cultural context from which the dominant values are derived is always in the background.

Business and civil society organizations influence ethics debates by employing moral arguments and leading information campaigns.

When it now comes to the development of a global TA, one can identify a number of parameters. It is important to mention that in a global system, parameters are not fixed as binaries (e.g. the existence or not of X). They refer to a continuum of innumerable points, whereby the focus is to identify how much of $X$ is there. With this in mind, the authors identified the following parameters that are necessary in the creation of a global TA [8]. Each of them represent a methodological concept that should be explored when applying a common TA:

The political systems range significantly, from multi-party to one-party systems, from liberal to authoritarian, from socialist to capitalist, from social welfare to free market. In addition, TA must deal with a basic question: Is TA possible in a non-liberal political system? The young and ongoing debate on this issue has already produced clear arguments on the negative, i.e. TA is not possible in an illiberal system [6], and also on the positive, i.e. TA or similar activities are possible in an illiberal system [17].

The normative foundation of TA has historically been a Western democratization project, and as such inherently democratic in its methods. Yet, it can be worthwhile to look for moral foundations of universal claims (such as the participation of public in S\&T decisions) in different contexts.

Public participation is one but not the only prerequisite for an "open system". Openness should be defined broader as the willingness to accept different perspectives, and part of this openness is to think about new TA methods.

The governance system of S\&T refers to the administrative set up around the decision making process. This is important in global TA as there are significant differences among countries that provide limitations and which need to be taken into consideration. For the development of global TA, the next governance level is more appropriate: global governance. Governance here refers to global decision making structures in S\&T.

S\&T priorities are closely connected to development needs such as water, food, housing etc. As a matter of fact, there is hardly a developing country in the world today that does not need to deal with both low and high technology developments at the same time. At the same time, a developed country could benefit from redirecting its focus to low-tech S\&T solutions, especially regarding sustainability aspects, such as energy needs. As such, there is an opportunity for significant developments in TA methodology at global level.

\section{CONCLUSIONS}

For the purpose of a global approach a wider view of ethics seems useful. This enables a way of understanding how ethical discourses shape S\&T policies and how science and technology are embedded in society. Further steps would be to understand deeper the interrelatedness of science and technology with a specific society and its values and then scaling this up to a global level. Already today, it is 
known that global platforms, capacity building and engagement are needed for any kind of mainstreaming while still accounting for specifics. For TA, one could think of a common online communication platform and a series of international TA conferences.

Scientific studies in the field of technology assessment are often concerned with the national context. Parliamentary TA with its clear addressee, is output oriented and aims to provide advice for decision making especially on a national level. Yet, in light of the grand challenges such as climate change, TA should reflect on how to possibly adapt its responses. This is not only a question of cooperation an effectiveness of the work. It's about the question how to provide advice about options in a global context and in which ways it can support an opening-up of discussions on technological futures. This should be continuously done in the national context but at the same time strive towards a global frame: "Instead of the old model of a country-based, government-led, 'glass and concrete' technology assessment office, a redesigned conceptualization of these activities has been proposed; one that is more transnational, networked, virtual and flexible than its predecessors, and crucially that combines citizen and decision-maker participation with traditional subject-matter-based expertise" [3]. What are the chances for a global TA after all? The opportunities for a common framework are clear if the community concentrates on the similarities and not just the differences: what risks for the environment and society do new technological developments entail? Or vice versa: What socio-technical innovations are needed to solve the great challenges of our time? As shown in this paper, there is an increasing requirement for TA to adapt to worldwide reach of S\&T and some challenges can only be met on a global level.

\section{REFERENCES}

[1] B. Bimber. The Politics of Expertise in Congress: The Rise and Fall of the Office of Technology Assessment. Albany: State University of New York Press, 1996.

[2] P.D. Blair. Congress's Own Think Tank. Learning from the Legacy of the Office of Technology Assessment (19721995). New York: Palgrave Macmillan, 2013.

[3] A. Ely, P. van Zwanenberg and A. Stirling. (2011). New Models of Technology Assessment for Development. STEPS Working Paper 45. [Online]. Available: http://steps-centre.org/wp-content/uploads/Technology_Assessment.pdf [Nov. 7, 2018].

\section{Constanze Scherz \\ Julia Hahn \\ Miltos Ladikas}

Institute for Technology Assessment and Systems Analysis (ITAS)

Karlsruhe Institute of Technology (KIT)

Karlstraße 11, 76133 Karlsruhe, Germany

e-mail: scherz@kit.edu

julia.hahn@kit.edu

miltos.ladikas@kit.edu
[4] J. Ganzevles and R. van Est. (2012). TA Practices in Europe. Deliverable 2.2 in the collaborative project on mobilization and mutual learning actions in European Parliamentary Technology Assessment. [Online]. Available: http://www.pacitaproject.eu/wp-content/uploads/2013/ 01/TA-Practices-in-Europe-final.pdf\%20 [Nov. 7, 2018].

[5] A. Grunwald. "Technology Assessment: Concepts and Methods," in Handbook of the Philosophy of Science: Philosophy of Technology and Engineering Sciences. A. Meijers, Ed. Amsterdam: Elsevier, 2009, pp. 1103-1146.

[6] A. Grunwald. „Technikfolgenabschätzung und Demokratie. Notwendige oder kontingente Verbindung?" TATUP Journal for Technology Assessment in Theory and Practice, vol. 27, pp. 40-45, 2018.

[7] D. Guston. "Insights from the Office of Technology Assessment and Other Assessment Experiences," in: Science and Technology Advice for Congress. M.G. Morgan and J.M. Peha, Ed. Washington, DC: Taylor \& Francis, 2003, pp. 7789.

[8] J. Hahn and M. Ladikas. Towards a Global Technology Assessment Approach. Karlsruhe: KIT Scientific Publishing, in print.

[9] J. Hahn, Chr. Merz and C. Scherz. "Identity Shaping. Challenges of Advising Parliaments and Society. A Brief History of Parliamentary Technology Assessment." Philosophy of Science and Technology, vol. 20, pp. 164-178, 2015.

[10] A. Irwin. "The Politics of Talk. Coming to Terms with the 'New' Scientific Governance." Social Studies of Science, vol. 36, pp. 299-320, 2006.

[11] S. Jasanoff. "Technologies of Humility: Citizen Participation in Governing Science." Minerva, vol. 41, pp. 223-244, 2003.

[12] A. Keiper. "Science and Congress." The New Atlantis, vol. 7, pp. 19-50, 2004.

[13] M. Ladikas and D. Schroeder. "Too Early for Global Ethics?" Cambridge Quarterly of Health Care Ethics, vol. 14, pp. 404-415, 2005.

[14] M. Ladikas, S. Chaturvedi, S., Y. Zhao and D. Stemerding. Science and Technology Governance and Ethics. A Global Perspective from Europe, India and China. Cham: Springer International Publishing, 2015.

[15] J. Sadowski and D. Guston. "Technology Assessment in the USA: Distributed Institutional Governance." TATUP - Journal for Technology Assessment in Theory and Practice, vol. 15, pp. 53-59, 2015.

[16] TAMI - Technology Assessment in Europe: Between Method and Impact. (2004). Final Report. [Online] Available: https://www.ta-swiss.ch/?redirect=getfile.php\&cmd[getfile][uid] $=944$ [Nov. 7, 2018].

[17] P.-H. Wong. "Responsible Innovation for Decent Nonliberal Peoples. A Dilemma?" Journal of Responsible Innovation, vol. 3, pp. 154-168, 2016. 